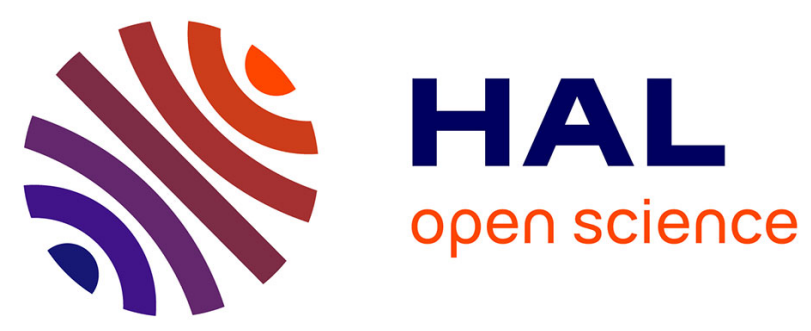

\title{
Penser les catégorisations sociales à l'aide de la linguistique interactionnelle
}

\author{
Clémence Léobal
}

\section{To cite this version:}

Clémence Léobal. Penser les catégorisations sociales à l'aide de la linguistique interactionnelle: A propos de MIGGE Bettina, LEGLISE Isabelle, Exploring Language in a Multilingual Context: Variation, Interaction and Ideology in Language Documentation, Cambridge University Press, New York, 2013.. Genèses. Sciences sociales et histoire, 2016, 102, pp.145-150. hal-01340244

\section{HAL Id: hal-01340244 \\ https://hal.science/hal-01340244}

Submitted on 1 Jul 2016

HAL is a multi-disciplinary open access archive for the deposit and dissemination of scientific research documents, whether they are published or not. The documents may come from teaching and research institutions in France or abroad, or from public or private research centers.
L'archive ouverte pluridisciplinaire HAL, est destinée au dépôt et à la diffusion de documents scientifiques de niveau recherche, publiés ou non, émanant des établissements d'enseignement et de recherche français ou étrangers, des laboratoires publics ou privés. 


\title{
Clémence Léobal (Cerlis/Iris)
}

\section{Penser les catégorisations sociales à l’aide de la linguistique interactionnelle.}

\author{
A propos de MIGGE Bettina, LEGLISE Isabelle, Exploring Language in a Multilingual \\ Context: Variation, Interaction and Ideology in Language Documentation, Cambridge \\ University Press, New York, 2013.
}

in Genèses, n²102, 2016, p.145-150.

Parmi les matériaux empiriques sur lesquels s’appuient les sciences sociales, les discours des acteurs figurent en bonne place. Toutefois, hormis la sociologie du langage ${ }^{1}$ et l'anthropologie linguistique, peu nombreux sont les travaux qui interrogent la langue dans laquelle ces discours sont produits. Pour les sociolinguistes, la langue est une idéalisation construite historiquement : ils s'intéressent aux conceptualisations réalisées par les locuteurs en fonction de ce qu'ils considèrent ou non comme des langues. Ce faisant, ces auteurs montrent que le questionnement sur les processus de construction des «langues » et sur les " pratiques langagières », est particulièrement riche d'enseignements pour aborder la question des catégorisations sociales, en liant les pratiques de dénominations et leurs usages dans l'interaction.

Exploring Language in a Multilingual Context porte sur une langue de Guyane communément appelée takitaki. Cette langue est utilisée dans la région du Bas-Maroni, située autour de la frontière franco-surinamaise, de part et d'autre du fleuve Maroni, aisément franchissable et abondamment traversé par les habitants. Cette région connaît des mutations sociales très rapides, du fait de l'exode rural, mais aussi des migrations régionales en provenance du Surinam, du Guyana, d'Haïti, du Brésil, d'Asie et d'Europe. Parlée des deux côtés de la frontière, l'entité linguistique appelée takitaki est pleinement liée à ces recompositions. Le takitaki est l'un des noms donnés en Guyane à des pratiques langagières rattachées aux créoles à base anglaise qui servent de lingua franca dans la région du Maroni.

En prenant pour objet des pratiques langagières au croisement de territoires et d'usages différenciés, le livre de Bettina Migge et Isabelle Léglise s’inscrit dans le champ d’étude des phénomènes de créolisation et de pidgnisation ${ }^{2}$, particulièrement révélateurs des processus de construction des langues. Pour mener ce projet à bien, l'ouvrage conjugue trois démarches inscrites dans la continuité de la sociolinguistique de Labov ${ }^{3,}$ qui prône de prendre le langage tel qu'il est effectivement produit dans son contexte social : démarche à la fois épilinguistique (sur les dénominations de langues), sociolinguistique (sur leurs usages et leurs

François Leimdorfer, Les sociologues et le langage. Paris, Editions de la MSH, 2011.

Robert Chaudenson fait une distinction entre créoles, idiomes issus des langues européennes et qui se sont formés dans les sociétés coloniales entre le XVIe et le XVIIIe siècle, et comportent des locuteurs natifs, et pidgins, qui n'ont pas de locuteurs natifs. Il rejette l'idée selon laquelle les créoles seraient des pidgins nativisés (ayant aquis des locuteurs natifs comme première langue) ou vernacularisés (fréquemment parlé). Robert Chaudenson, La créolisation: théorie, applications, implications, Paris, Harmattan, 2003.

William Labov, Sociolinguistique [Sociolinguistic patterns, 1972]. Paris, Editions de Minuit, 1976. 
représentations), et de linguistique descriptive. Les auteures - toutes deux sociolinguistes, et engagées dans des collaborations scientifiques sur le terrain guyanais depuis longtemps ${ }^{4}$ mobilisent ici la sociolinguistique des idéologies de John Gumperz ${ }^{5}$, qui articule les visions des individus sur la langue, les processus sociaux et les pratiques linguistiques elles-mêmes. Elles explorent à la fois la question de la dénomination de la langue (qui emploie cette catégorie de takitaki et pourquoi?), celle de ses usages en situation (quand emploie-t-on le takitaki et comment ?) et enfin s'attachent à décrire la structure linguistique de cette entité (qu'est-ce que le takitaki?).

En prenant pour objet le takitaki, Exploring Langage in a Multilingual Context fait exception dans la littérature scientifique régionale qui rejette cette dénomination, supposée péjorative, exogène et imprécise. Cette expression signifie littéralement "bavardages », "ragôts », ou « propos nuisibles », ou encore «faire des histoires », « faire du bruit » ou «parler pour ne rien dire ». Les chercheurs préfèrent d'autres qualificatifs désignant les langues de cette famille, tels que sranan tongo (le créole surinamais), nenge(e) tongo, la langue des groupes marrons originaires du Haut-Maroni, ou encore ndjuka, aluku ou pamaka, langues parlées par chacun de ces groupes marrons éponymes. Dans leurs premiers travaux, les deux auteures partageaient ce positionnement ${ }^{6}$. Toutefois, l'expérience du terrain urbain, à Saint-Laurent-duMaroni, où elles ont fréquemment rencontré des personnes affirmant parler le takitaki, les a amenées à prendre des distances avec les catégories des textes scientifiques, eux-mêmes partie prenante des processus de dénomination. D’une manière originale dans le champ des études sur la créolisation, elles ont décidé de faire de ce phénomène du takitaki leur objet de recherche, abandonnant la posture normative condamnant l'usage de ce terme. Elles ont donc collecté des déclarations de pratiques lors d'une enquête menée dans les écoles par questionnaires; des interviews avec des habitants autour des dénominations; un corpus enregistré de conversations ; des enregistrements de propos bilingues ; enfin, des données ethnographiques collectées dans des villages pamaka du Moyen-Maroni et dans la ville de Saint-Laurent-du-Maroni.

La première partie de l'ouvrage est consacrée à une réflexion sur les dénominations de la langue. Elle montre comment les conceptualisations indigènes des pratiques langagières sont liées à des positions sociales. Elles s'inscrivent dans le courant de l'anthropologie du langage sur les noms de langue ${ }^{7}$, soulignant que la polynomie est la règle plutôt que l'exception ${ }^{8}$. Ainsi, les mêmes propos tenus pourront être qualifiés de ndjuka, d'aluku ou de takitaki en fonction du contexte. 
Comme toute langue, le takitaki n’est qu'un terme générique désignant un phénomène sociolinguistique complexe. Les auteures ne présupposent pas qu'il s'agisse d' " une entité monolithique avec une nature linguistique singulière, associé à une communauté de locuteurs bien définie et à une fonction sociale unique ${ }^{9}$ (p.308). Elles mobilisent le concept de language ideologies, défini par l'anthropologie linguistique états-unienne comme des croyances ou sentiments à propos des langues telles qu'elles sont pratiquées dans leur monde social : ces idéologies sont plurielles, construites dans l'intérêt d'un groupe, et utilisées dans la construction des identifications, de manière plus ou moins consciente ${ }^{10}$. Les chercheuses ne cherchent toutefois pas à savoir « de qui » c'est la langue, refusant l’idéologie nationaliste « 1 peuple, 1 langue, 1 nation », mais se contentent de définir plus prosaïquement qui la parle. Cela leur permet de constater que loin d'être parlé par les seuls Marrons du Maroni, fréquemment associés à cette entité, le takitaki est parlé par divers groupes, à commencer par les Amérindiens qui le parlent en première langue. Les auteures prennent également en considération les personnes qui parlent takitaki en seconde langue ou plus, dans un contexte multilingue : les migrants des autres parties du Surinam, dont les Marrons saamaka; de la grande région (Guyana, Caraïbes), de Chine, ou encore les français métropolitains et les créoles venus travailler dans l'Ouest guyanais. Le takitaki est donc la langue parlée par un ensemble multiforme de personnes déclarant le parler, composé de classes populaires pour qui le takitaki est la langue véhiculaire du quotidien, et de classes moyennes francophones qui apprennent le takitaki pour parler à des patients, élèves ou usagers.

Les trajectoires des locuteurs éclairent les pratiques de dénomination, qui varient selon qu'ils considèrent le takitaki comme une langue principale, comme une langue seconde, ou comme une langue étrangère. Ainsi, les jeunes Marrons, en affirmant parler le takitaki, veulent se distinguer des générations précédentes, associées à la ruralité, et affirmer une forme d'urbanité marronne sophistiquée, commune à tous les groupes marrons. Un Amérindien peut au contraire affirmer parler un takitaki différent des Marrons, dans un souci de distinction. Les individus de classe moyenne emploient la catégorie de takitaki à la fois pour affirmer leur ancrage local, dévaloriser les créoles à base anglaise supposés particulièrement " faciles » à apprendre car simplifiés, ou affirmer leur supériorité sur les classes populaires. Le caractère vague de cette entité takitaki permet en outre, pour un même individu, de l'utiliser à des fins diverses, sans avoir à les spécifier : l'interprétation est laissée à l'interlocuteur. Les différents usages du terme takitaki permettent de redessiner des frontières, mais aussi de produire de nouvelles réalités.

Les auteures s'attachent dans un second temps à décrire la réalité des pratiques langagières désignées sous cette étiquette, s'appuyant sur une description de cette forme linguistique en tant que telle. Différentes conceptualisations de cette entité se chevauchent mais ne se recoupent pas tout-à-fait: il s'agirait d'un mélange lexical, combinant des caractéristiques de l'aluku, du ndjuka et du pamaka (langues parlées par les groupes marrons du Maroni éponymes), et du sranan tongo (créole surinamais). 
Bettina Migge et Isabelle Léglise s’appuient sur des enregistrements, réalisés par elles-mêmes et par des enquêteurs locuteurs du takitaki, et sur des entretiens réalisés avec des individus issus des différents groupes de locuteurs, et de situations d'interaction quotidiennes. Le takitaki se caractérise par une grammaire simplifiée par rapport aux variétés parlées par les Marrons dans les contextes endolingues (sans contact de langues), parfois mélangée avec les grammaires des langues d'origine des locuteurs. On assiste donc à un processus de koinéisation : ce concept forgé par Siegel (1985), à propos du Hindi, renvoie au mélange de traits de différents dialectes régionaux apparentés, qui sont nivelés et réduits dans le contexte de la migration et du contact entre locuteurs de différentes variétés (Siegel 1997) ${ }^{11}$. Le takitaki a ceci de spécifique de ne pas avoir été créé en interne au groupe des locuteurs, mais de manière exolingue (en contact avec d'autres langues).

Les auteures ne se limitent pas aux caractéristiques linguistiques, mais examinent aussi les interactions. Elles mettent en évidence que la koinéisation des pratiques langagières découle des pratiques des locuteurs natifs eux-mêmes. D'un côté, ils la simplifient pour mieux se faire comprendre d'autres locuteurs, ce qui pourrait être à l'origine de l'idée, répandue dans la classe moyenne, que le takitaki n'est pas une vraie langue et manque de complexité. De l'autre, ils pratiquent des alternances codiques (code-switching). Le takitaki est ainsi caractérisé par l'alternance codique : les enregistrements comportent des variations internes, introduisant du lexique de langues marronnes, du sranan tongo (créole surinamais), voire du français, anglais et hollandais. Les locuteurs plurilingues intercalent des mots ou des phrases issus de divers codes. Alors que les premiers travaux sur les alternances codiques s'intéressaient aux situations de bilinguisme, par exemple dans les communautés hispanophones des Etats-Unis ${ }^{12}$, ces pratiques relèvent aussi, pour les auteures, d'une manière de s'affirmer au monde, sans être nécessairement bilingue. Les choix entre codes dépendent des interlocuteurs, de leur trajectoire, de leur usage de la langue, ainsi que de la situation d'interaction. Ces alternances sont signifiantes, et sous-tendent des affichages d'identité. Le vocabulaire du sranan tongo peut être utilisé par exemple pour parler de travail salarié, par opposition à l'agriculture vivrière, évoquée par un lexique tiré des langues marronnes. Elles peuvent aussi avoir une signification dans l'interaction précise : l'usage du sranan tongo peut marquer un agacement, une supériorité sur l'interlocuteur. Les pratiques d'alternance codique caractérisant le takitaki relèvent donc d'enjeux discursifs et expressifs, qui ne sont pas toujours perçus de la même manière par les interlocuteurs.

Considérant qu'il y a en général au moins un Marron dans l'interaction - et laissant de côté les autres native speakers que sont les Amérindiens -, les auteures étudient des interactions entre Marrons et non-Marrons. Elles vont d'interactions basiques, lors desquelles des locuteurs de classe moyenne comprennent approximativement les propos, pourtant simplifiés, des locuteurs marrons (par exemple entre un médecin et ses patients), à des interactions plus fluides, où les Marrons simplifient leur langue et y mettent beaucoup de lexique du sranan and pidgin/creole development », in Arthur K. Spears et Donald Winford (dir), The Structure and Status of Pidgins and Creoles, ,Amsterdam, John Benjamins, 1997, pp. 111-149.

12 John Gumperz, Engager la conversation. Introduction à la sociolinguistique interactionnelle. Paris, Les éditions de minuit, 1989. 
tongo, dans le but de paraître urbains. Ces enjeux discursifs ne sont pas toujours perçus par les locuteurs non-natifs, qui y voient des pratiques langagières équivalentes les unes aux autres, d'où le nivellement des pratiques. Les Marrons en contexte urbain disséminent donc activement des pratiques nouvelles, comme lors des salutations où ils découragent les nonMarrons de les faire à l'ancienne ${ }^{13}$.

Dans les interactions entre Marrons urbains, le takitaki correspond à une pratique langagière distincte des langues marronnes du Maroni : elle dénote une position urbaine, moins marquée ethniquement que les langues marronnes. Même s'il existe des distinctions entre les pratiques des jeunes Marrons, marquées par un fort usage du lexique des langues marronnes, et celles des autres locuteurs, leurs pratiques tendent à se ressembler en surface: ce sont ces ressemblances que la dénomination takitaki semble vouloir mettre en avant.

Ainsi, cette étude des pratiques langagières permet de comprendre comment des conceptualisations différentes de ce qu'est le takitaki à la fois reflètent et participent à produire des pratiques langagières quotidiennes très largement partagées dans le Bas-Maroni. Les auteures élargissant la notion d'émique au-delà des seuls native speakers: on peut rapprocher cette démarche de celle de Max Gluckman, qui analysait la société du Zululand comme un tout incluant Zulus et colons ${ }^{14}$. Cela leur permet de sortir des seules analyses centrées sur les rapports interethniques : la construction du savoir par ethnie ne permet pas de comprendre les processus de changements en contexte urbain. Elles mettent en lumière des processus habituellement invisibilisés : la pratique du takitaki étant commune à des groupes variés, son étude permet de décrire des hiérarchies de classe, mais aussi de faire des distinctions internes aux groupes ethniques, notamment entre Marrons "anciens et ruraux" d'une part, "jeunes et urbanisés" de l'autre.

Par ailleurs, ce livre offre une réflexion sur les dénominations des langues en les rattachant aux usages de ces catégorisations et aux pratiques langagières elles-mêmes : cette démarche sociolinguistique montre comment les catégorisations sont produites par les usages que les acteurs en font. Ce questionnement sur les catégories appliquées aux langues gagnerait à être élargi à tous les processus de catégorisation. L'ouvrage, dont les premiers chapitres retracent l'histoire des Marrons, n'étend pas le questionnement sur les catégorisations des langues à celui des catégorisations ethniques. Le lien entre pratiques langagières et ethnicité est pourtant à examiner avec prudence, notamment quand il conduit à affirmer, par exemple, que $60 \%$ de la population de l'Ouest de la Guyane est d'origine marronne (p.11). Cette affirmation repose peut-être sur leur enquête effectuée à l'école, qui recensait environ $60 \%$ d'enfants déclarant parler des langues marronnes en langue maternelle. L'équivalence que font les auteures avec une identification ethnique supposée marronne ne va pourtant pas de soi.

Au niveau méthodologique, on peut regretter que les transcriptions d'enregistrements ne soient pas rattachées aux trajectoires des locuteurs, introduites dans un chapitre préalable et

\footnotetext{
13 Bettina Migge, "Greeting and social change », in Susanne Mühleisen, et Bettina Migge (dir), Politeness and Face in Caribbean Creoles, Amsterdam, John Benjamins, 2005.

14 Max Gluckman, «Analysis of a social situation in modern Zululand » [1940], Genèses n 72, 2008, pp. 119-55. Traduit et commenté par Yann Tholoniat et Benoît de L’Estoile.
} 
séparé. La prise en compte des positions sociales dans l'analyse des interactions est donc réduite à l'appartenance à quatre groupes : la classe moyenne française ; les migrants de la grande région; les Marrons du Maroni ; et les Amérindiens. Une analyse appuyée sur les trajectoires individuelles des locuteurs aurait pu permettre de déconstruire davantage ces appartenances, réduites à ces groupes établis par les chercheures - même s si elles innovent en combinant ethnicité, classe et origine géographique. Néanmoins, il importerait de prendre en compte ces identifications ethniques comme constructions langagières fluides et mouvantes. L'argumentation des auteures opère en outre un glissement : la notion de native speaker finit par être assimilée aux seuls Marrons du Maroni, excluant notamment les Amérindiens. Or la distinction entre Marrons et non-Marrons semble relever des mêmes processus complexes de chevauchements entre assignations identitaires et appartenances mis en évidence à propos des dénominations de langue. Il semblerait donc fécond d'allier les apports de l'analyse des pratiques langagières développée en sociolinguistique avec les réflexions sur les catégorisations en sciences sociales.

Clémence Léobal est doctorante en sociologie à l'Université Paris-Descartes (Cerlis), et à l'Iris (Ehess). Sa thèse porte sur les modes d'habiter et politiques urbaines en contexte postcolonial et transfrontalier (Saint-Laurent-du-Maroni, Guyane). Elle a récemment publié Saint-Laurentdu-Maroni: Une porte sur le fleuve aux Editions Ibis Rouge, 2013. 